\title{
Hybrid model for movie recommendation system using content K-nearest neighbors and restricted Boltzmann machine
}

\author{
Dayal Kumar Behera1, Madhabananda Das ${ }^{2}$, Subhra Swetanisha ${ }^{3}$, Prabira Kumar Sethy \\ ${ }^{1,2}$ School of Computer Engineering, KIIT Deemed to be University, Bhubaneswar, India \\ ${ }^{3}$ Department of CSE, Trident Academy of Technology, Bhubaneswar, India \\ ${ }^{4}$ Department of Electronics, Sambalpur University, Jyoti Vihar, Burla, Odisha India
}

\begin{tabular}{l} 
Article Info \\
\hline Article history: \\
Received Mar 16, 2021 \\
Revised Jun 3, 2021 \\
Accepted Jun 17, 2021 \\
\hline Keywords: \\
Collaborative filtering \\
Content KNN \\
Movie recommendation \\
RBM \\
Recommender system
\end{tabular}

\begin{abstract}
One of the most commonly used techniques in the recommendation framework is collaborative filtering (CF). It performs better with sufficient records of user rating but is not good in sparse data. Content-based filtering works well in the sparse dataset as it finds the similarity between movies by using attributes of the movies. RBM is an energy-based model serving as a backbone of deep learning and performs well in rating prediction. However, the rating prediction is not preferable by a single model. The hybrid model achieves better results by integrating the results of more than one model. This paper analyses the weighted hybrid CF system by integrating content $\mathrm{K}$ nearest neighbors (KNN) with restricted Boltzmann machine (RBM). Movies are recommended to the active user in the proposed system by integrating the effects of both content-based and collaborative filtering. Model efficacy was tested with MovieLens benchmark datasets.
\end{abstract}

This is an open access article under the CC BY-SA license.

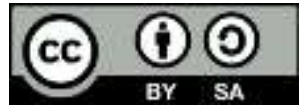

\section{Corresponding Author:}

Prabira Kumar Sethy

Department of Electronics

Sambalpur University

Jyoti Vihar, Burla, Odisha India

Email: prabirsethy.05@gmail.com

\section{INTRODUCTION}

Recommender system [1] is preferable in the era of information overload to help users discover interesting things from an extensive collection of data. Collaborative filtering (CF) [2], [3] algorithms are commonly used to create recommender systems because they efficiently use the users' rating information. An item rating matrix can be used to explore other invisible or unfound items for similar users. Hybrid RS combines CF recommendation and content-based RS. The CF-based recommendation is applicable in many application domains [4], [5]. Model-based Collaborative filtering approach uses many machine learning [6] based models such as Random forest [7], support vector machine (SVM) [8], and matrix factorization [9] for predicting the users' likeness. It is estimated that many streaming services companies create a lot of revenue by applying movie recommendation techniques. Various models have been proposed to improve the productivity of the movie recommendation [10] framework.

CF methods are broadly divided into two categories: memory-based and model-based. In a sparse data set, the memory-based model does not perform well, unlike the model-based CF. K-nearest neighbors [11]-[13] based model is successfully applied in many classification models. RBM [14] has attained much attention in recent CF literature, as it has a compatible graphical structure with SVD and PMF. Salakhutdinov et al. [15] demonstrated the use of RBM in CF and scored 6 percent higher than the standard baseline predictor system of Netflix. It also serves as building blocks for DBN architecture [16] and many other deep learning [17]-[19] models. 
Chunchun $\mathrm{Li}$ and Jun $\mathrm{Li}$ [20] have used dual cRBM to address dual user and item patterns. In their model rating matrix reflects a latent matrix of features. They used separate RBM for each user, proposing a restricted RBM (cRBM) recommendation for Netflix movies.

The Content-based recommendation focuses primarily on movie description or details, while CF focuses on user ratings for the movie. If rating information is considered, it is possible to collect extremely sparse data as few consumers choose to give the rating of the product after purchase. RBM, along with Content KNN, is used to address this issue. Fischer and Igel [21] discussed different RBM learning algorithms such as CD and parallel tempering. The modified CD algorithm called 'Lean Contrastive Divergence' was proposed by Ning et al. [22] to increase the speed of the learning and prediction process. Pujahari et al. [23] proposed a CF model by taking the preference relationship as an input to RBM and by combining the side information of the movies, trying to generate the ranking of the items. Kilani et al. suggested a hybrid RS [24] based genetic algorithm using MF and KNN that uses both latent factor and neighborhood data to recommend items to an active user. In literature it is shown that ensemble models [25], [26] works better in text-based analytics [27]-[29], intrusion detection[30] and in classification of sounds signals [31].

The content-based KNN works well by finding similarities between movies based on the movie's descriptions and other attributes. It does not depend on the users' rating. In comparison to the CF model [32], RBM performs well in large databases. Contributions of this work are stated as below:

- Studying the impact of various similarity measures in user-based and item-based collaborative filtering.

- Designing a recommender system to deal with the sparse dataset by using the movies' profile such as year of release, genre, and description features.

- Designing a model-based recommender system using Restricted Boltzmann Machine.

- Proposing a weighted hybrid model based on content KNN and RBM for capturing both content-based filtering and higher-order model-based collaborative filtering.

\section{RESEARCH METHOD}

Consider $U$ as the consumer vector; and $\mathrm{M}$ as the movie vector where $\mathrm{p}, \mathrm{q}$ is the user and movie numbers, respectively. $\mathrm{R}$ is the matrix with the form $\mathrm{p} \times \mathrm{q} . \mathrm{r}_{\mathrm{um}}, \hat{\mathrm{r}}_{\mathrm{um}}$ denotes the real rating of the consumer ' $u$ ' for the movie ' $m$ ' and the expected ratings. The similarity between user ' $u$ ' and user ' $v$ ' can be calculated using (1) [33]. $\bar{r}_{u}$ and $\bar{r}_{v}$ represents the average rating of user ' $u$ ' and user ' $v$ ', respectively. Rating of user ' $u$ ' for movie ' $\mathrm{m}$ ' can be calculated using (5).

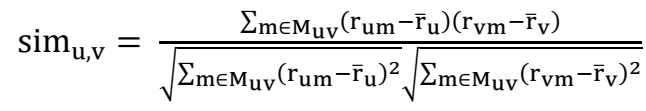

$$
\begin{aligned}
& \mathrm{M}_{\mathrm{uv}}=\mathrm{M}_{\mathrm{u}} \cap \mathrm{M}_{\mathrm{v}} \\
& \bar{r}_{u}=\frac{\sum m \in M_{u v} r_{u m}}{\left|M_{u v}\right|} \\
& \overline{\mathrm{r}}_{\mathrm{v}}=\frac{\sum \mathrm{m} \in \mathrm{M}_{\mathrm{uv}} \mathrm{r}_{\mathrm{vm}}}{\left|\mathrm{M}_{\mathrm{uv}}\right|} \\
& \hat{\mathrm{r}}_{\mathrm{um}}=\mathrm{b}_{\mathrm{um}}+\frac{\sum_{\mathrm{v} \in \mathrm{N}_{\mathrm{m}}^{\mathrm{k}}(\mathrm{u})} \operatorname{sim}_{\mathrm{u}, \mathrm{v}} \cdot\left(\mathrm{r}_{\mathrm{vm}}-\mathrm{b}_{\mathrm{vm}}\right)}{\sum_{\mathrm{v} \in \mathrm{N}_{\mathrm{m}}^{\mathrm{k}}(\mathrm{u})} \operatorname{sim}_{\mathrm{u}, \mathrm{v}}}
\end{aligned}
$$

$b_{u m}=\mu+b_{u}+b_{m}$ is $u$ 's baseline estimate [34] for movie $m$.

The parameters $b_{u}$ and $b_{m}$ indicate user ' $u$ ' and movie ' $m$ ' rating deviations. Generally, stochastic gradient descent (SGD) or alternating least squares (ALS) algorithm solves these parameters. To find similarities between movies ' $x$ ' and ' $y$ ', modified cosine-similarity measure in (6) is used.

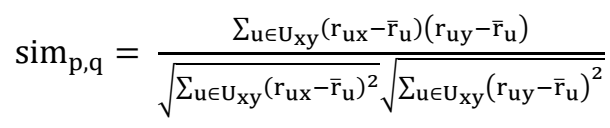

The problem with this baseline is that to find similarity between user ' $u$ ' and ' $v$ ', the ratings provided by user's ' $u$ ' and ' $v$ ' are taken into account. It does not fit well in sparse data. 


\subsection{RBM}

RBM is an energy-based, probabilistic model with only two layers of the neural network structure: a hidden layer and a visible layer. This two-layer network has the restriction that there is no connection between two nodes in the same layer. It is commonly used in collaborative filtering to learn the distribution of probability over a ranking matrix. This network is trained through gradient descent and backward propagation, where each iteration is having a forward pass and backward pass (reconstruction). Information exchange between neurons in the same layer is limited in RBM. There is a relation between two separate layers of neurons. RBM is trained in forward and backward passes, and the inputs are rebuilt in the backward pass. This is achieved over several epochs before it converges on a set of weights and distortions that minimizes the reconstruction error.

It describes a distribution over $\mathrm{V}$ with a layer of binary secret unit ' $\mathrm{h}$ '. The input layer $\mathrm{V}$ is a matrix of $\mathrm{k} \times \mathrm{m}$ dimension and $V_{p}^{k}=1$, if user rating for the movie ' $\mathrm{m}$ ' is ' $\mathrm{k}$ '. The likelihood of each visible/input binary matrix V column is modeled with the distribution "Softmax." Hidden user functions ' $h$ ' is likely to be modeled with sigmoid function. The energy of the state $(V, h)$ is given in (7) [21].

$$
E(V, h)=-a^{T} V-b^{T} h W-h^{T} W V
$$

In the learning process, the training vector V's log-likelihood to weight can be determined using (8).

$$
\frac{\partial \log \log \operatorname{prob}(V)}{\partial W_{p q}}=\left\langle\vartheta_{p} h_{q}\right\rangle_{d a t a}-\left\langle\vartheta_{p}, h_{q}\right\rangle_{\text {model }}
$$

Where, $\left\langle\vartheta_{p}, h_{q}\right\rangle_{\text {model }}$ is the expectation of the distribution whose complexity is very high. In [6], CD (Contrastive Divergence) is used to estimate expectation. The weight can be modified using stochastic ascent given in (9).

$$
\Delta w_{p q}=\alpha\left(\left\langle\vartheta_{p} h_{q}\right\rangle_{d a t a}-\left\langle\vartheta_{p}, h_{q}\right\rangle_{\text {model }}\right)
$$

Where, $\alpha$ is the learning rate. To overcome the complexity, we have used 1-step CD and the values are updated using (10).

$$
\Delta \mathrm{w}_{\mathrm{pq}}=\alpha\left(\left\langle\vartheta_{\mathrm{p}} \mathrm{h}_{\mathrm{q}}\right\rangle_{\text {data }}-\left\langle\vartheta_{\mathrm{p}}, \mathrm{h}_{\mathrm{q}}\right\rangle_{\text {reconstruction }}\right)
$$

In the proposed model, a hybrid model is considered with equal weightage for both RBM and content KNN model. Figure 1 depicts the training of the hybrid model.

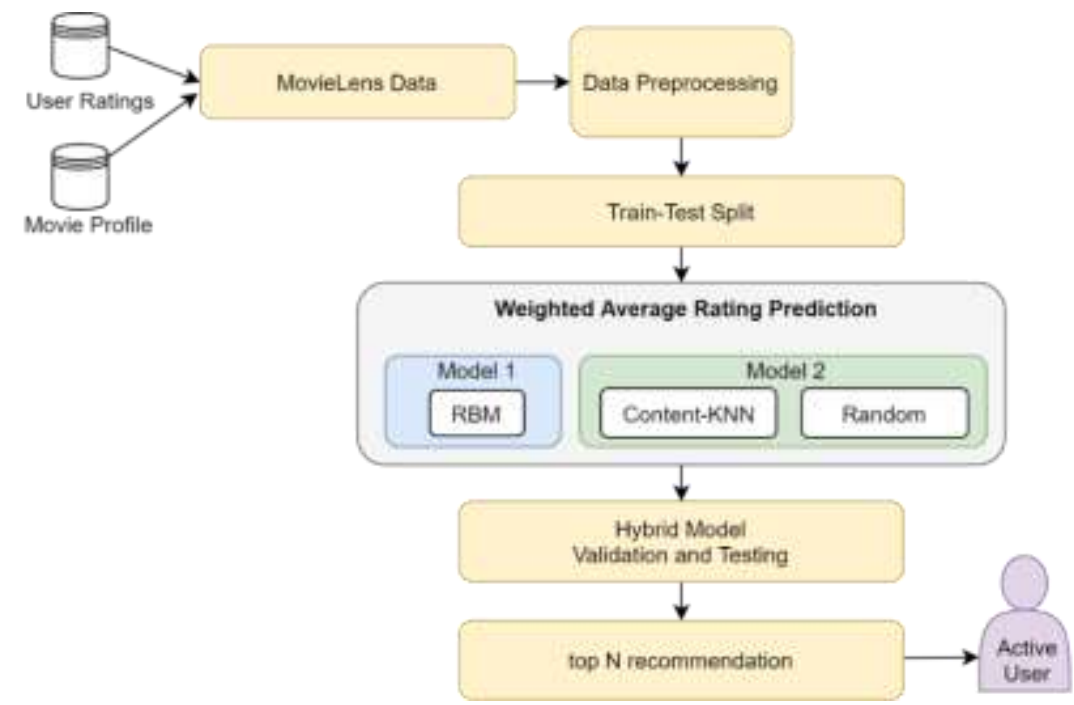

Figure 1. The proposed hybrid model 
On the MovieLens dataset, our proposed model is implemented. This dataset contains user interests and movie profiles. This data collection is then subjected to preprocessing of data, in which some samples of data are obtained. The 70-30 test division is imposed on the preprocessed data. In this step of estimation, the RBM is used, and the RBM is combined with the content KNN. Content KNN finds the similarity between different movies based on attributes of movies such as year of release, genre, box office hit rate, and descriptions of the movie. The weighted average is obtained from the above model, and the validated model is used to produce a top $\mathrm{N}$ recommendation for the active user.

\section{RESULTS AND DISCUSSION}

In python 3.8, the above designed algorithms have been implemented, and NumPy, pandas, surprise are the main libraries used for implementation. RBM network is trained in the forward pass by feeding training information on the visible layer and training weights and biases between them during the backward pass. To generate the output of any hidden neuron, an activation function rectified linear units (ReLU) [35] has been used. ReLU is preferred here as it does not activate all the neurons at the same time.

For the model training, each user's rating from the training set is passed as a batch into the RBM. The nodes in the visible layer reflect the user's ratings on each movie. Interconnection weights are learned to reconstruct the ratings for user-movie pairs that are missing. Here, each individual rating is treated as five visible nodes, one for each possible rating value. Rating in the RBM of five movies having ratings 5, NA, 3, NA and 2, respectively, is shown in Figure 2. Rating of first movie is 5 , hence $5^{\text {th }}$ column is set to 1 . Similarly, column 2 of movie 5 is set to 1 .

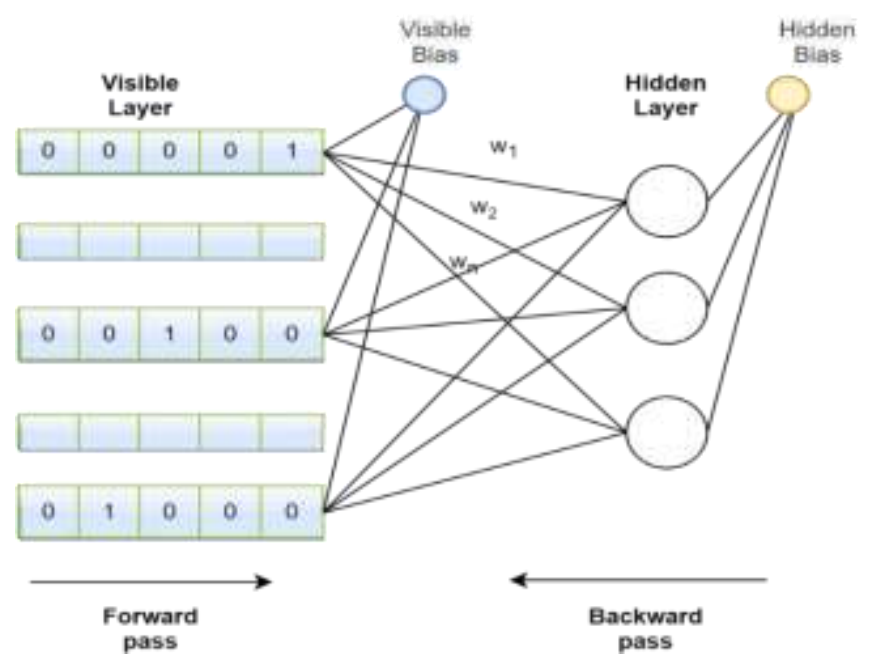

Figure 2. Representation of rating information in RBM

There is a need to estimate missing ratings for movies 2 and 4 . The mutual weight and bias can be used to predict the missing score once the model is trained. Many scores are missing in the real dataset. Instead of training on any possible mix of users and movies, the model is trained on the available data by removing the missing scores. In general, using contrastive divergence and Gibb's sampling, the model samples the probability distribution. The resulting weights and biases are reused for other users after the model has been trained for one user.

The number of visible nodes is calculated as the product of number of movies and rating values given by the user. In the dataset ML100K, the number of movies is 8211 , and number of users in the train set is 671 . So, the maximum number of ratings possible for the user is $8211 * 5=41055$ and the training rating matrix dimension is $671 \times 41055$. The hidden node represents the latent variable. The numbers of hidden node are taken 50 for the experiment. Epochs represent the number of iterations to take for the forward and backward pass. In each epoch, to minimize the error between actual and reconstructed rating value, the model will be trained across all the users in the train set. The number of epochs is set to 5 to 30 with an increment of 5. The impact of the epochs in the performance metrics is shown in Figure 3. The learning rate controls how quickly the error converges and it is set to 0.001. Batch size controls the number of users to be processed at a time, and it is set to 100 . Once the model is trained, the updated weight and biases are used to get the 
recommendation for the active user. The softmax activation function is used in the visible layer to get the probability score that indicated the likeness of the user for a product.

$\mathrm{RBM}$ is combined with Content KNN to consider the advantages of both content-based filtering and model-based collaborative filtering model. Content KNN works well even for new users and RBM works well for large user ratings. The performance of various ensemble models has been evaluated on MovieLens1M and 100K datasets downloaded from the grouplens.org site. $70 \%$ data is used for training the model and $30 \%$ data is used to test the model.

Figure 4 depicts the results of User-based and Item-based CF under various similarity measures such as cosine, pearson, and mean squared difference (MSD). Item-based CF performs better under MSD similarity. In Content KNN, by finding the similarity based on year of release and genre, RMSE scores in ML100K dataset are 1.0624 and 1.0548, respectively. It indicates that genre plays a major role in recommending the movies. Accuracy measure is not considered here, as the model is not a classification model.

Results of combining RBM with Content KNN are shown in Table 1. w1 and w2 represent weight assigned to RBM and content KNN, respectively. A series of experiments are performed to see the impact of $\mathrm{w} 1$ and $\mathrm{w} 2$. To generate the rating prediction of the target user-movie pair, a weighted average is computed by considering w1 and w2 weights of the models. Root mean square error (RMSE), mean absolute error (MAE) [36] scores of the hybrid algorithms are shown in Table 1. A lower value indicates better results. ML1M, ML100K represents MovieLens one million and 100K ratings, respectively. For content KNN, the K value is set to 40. Figure 3 and Table 2 illustrate the RMSE score of various algorithms in different epochs of training RBM. The average value of RMSE is calculated in the Random model for different epochs.

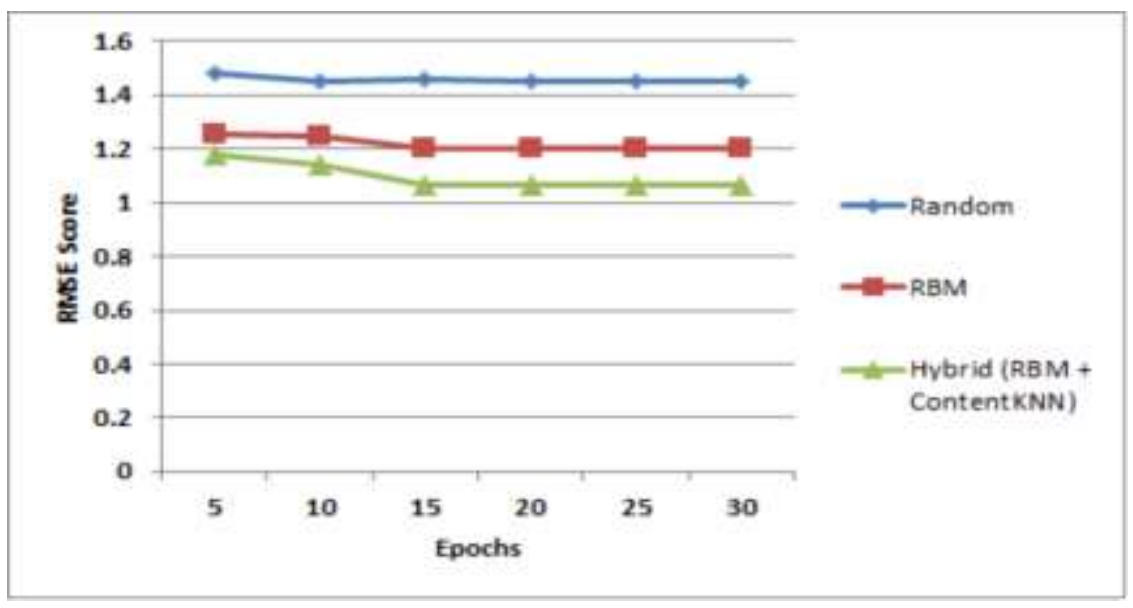

Figure 3. RMSE score of various models on ML1M data

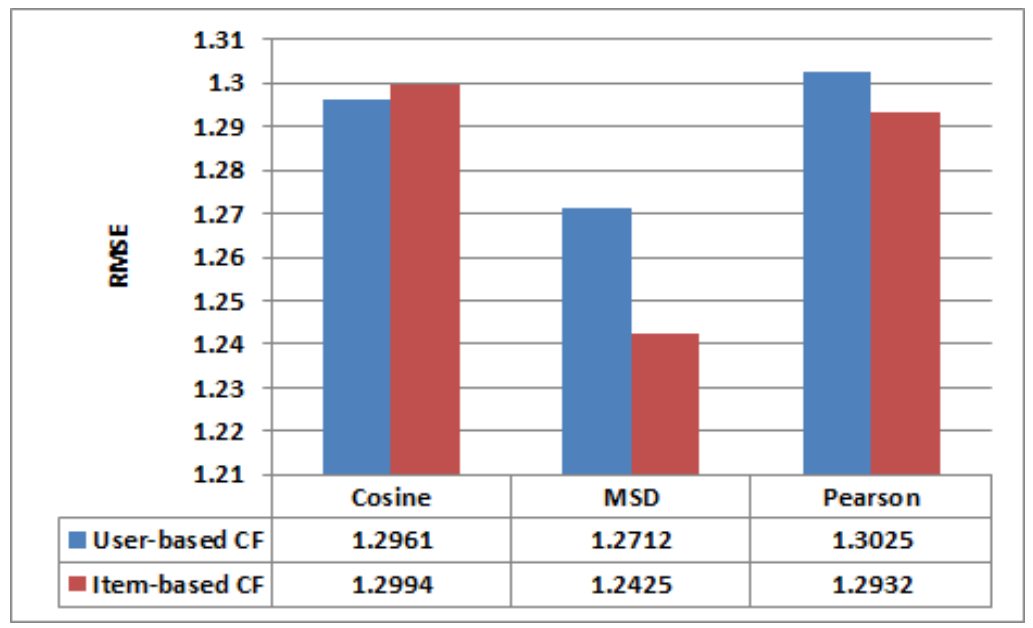

Figure 4. RMSE score of User-based and Item-based CF 
Table 1. Performance metrics of RBM and Hybrid models on MovieLens data

\begin{tabular}{lcccc}
\hline \multicolumn{1}{c}{ Dataset } & \multicolumn{2}{c}{ RMSE } & \multicolumn{2}{c}{ MAE } \\
\hline Random & 1.5067 & 1.4615 & 1.20 & 1.16 \\
RBM & 1.2126 & 1.1985 & 0.96 & 0.86 \\
ContentKNN & 1.0548 & 1.0322 & 0.74 & 0.72 \\
RBM+ContentKNN & 1.1155 & 1.0652 & 0.93 & 0.76 \\
RBM + Random & 1.3254 & 1.2578 & 1.05 & 0.98 \\
\hline
\end{tabular}

Table 2. RMSE score of various models on ML1M data

\begin{tabular}{|c|c|c|c|c|c|c|}
\hline Epochs & 5 & 10 & 15 & 20 & 25 & 30 \\
\hline Ran & 1.4821 & 1.4547 & 1.4615 & 1.4505 & 1.4505 & 1.4505 \\
\hline RBM & 1.2513 & 1.2466 & 1.1985 & 1.1985 & 1.1985 & 1.1985 \\
\hline Hybrid (RBM + ContentKNN) & 1.1812 & 1.1406 & 1.0652 & 1.0652 & 1.0652 & 1.0652 \\
\hline
\end{tabular}

\section{CONCLUSION}

Collaborative filtering and content-based recommendation are the popular techniques in filtering movies from a large collection. In CF, the mean square difference performs better as compared to cosine and Pearson similarity measures. In content KNN, genre-based calculation highly impacts the recommendation. RBM fits well in model-based collaborative filtering and content KNN in very sparse dataset. The goal of this paper is to use a weighted hybrid model to see both RBM and content KNN's combined impact on the movie recommendation. Both the models are assigned with equal weightage. The effect of the hybrid model on rating data from MovieLens $100 \mathrm{~K}$ and $1 \mathrm{M}$ is thoroughly investigated. Table 1 displays the RMSE and MAE score of different models. RBM with content KNN is shown to have better efficiency. Random movie recommendation is the worst performer. In the future, RBM and content KNN can be extended to the fields of teaching, music, news, and other recommendations.

\section{REFERENCES}

[1] V. Agarwal and A. Vijayalakshmi, "Recommender system for surplus stock clearance," International Journal of Electrical and Computer Engineering, vol. 9, no. 5, pp. 3813-3821, 2019, doi: 10.11591/ijece.v9i5.pp3813-3821.

[2] N. S. A. Rahman, L. Handayani, M. S. Othman, W. M. Al-Rahmi, S. Kasim, and T. Sutikno, "Social media for collaborative learning," International Journal of Electrical and Computer Engineering, vol. 10, no. 1, pp. 1070-1078, 2020, doi: 10.11591/ijece.v10i1.pp1070-1078.

[3] S. Babeetha, B. Muruganantham, S. Ganesh Kumar, and A. Murugan, "An enhanced kernel weighted collaborative recommended system to alleviate sparsity," International Journal of Electrical and Computer Engineering, vol. 10, no. 1, pp. 447-454, 2020, doi: 10.11591/ijece.v10i1.pp447-454.

[4] M. Jalili, S. Ahmadian, M. Izadi, P. Moradi, and M. Salehi, "Evaluating Collaborative Filtering Recommender Algorithms: A Survey," IEEE Access, vol. 6, pp. 74003-74024, 2018, doi: 10.1109/ACCESS.2018.2883742.

[5] D. K. Behera, M. Das, and S. Swetanisha, "A Research on Collaborative Filtering Based Movie Recommendations: From Neighborhood to Deep Learning Based System," Int. J. Recent Technol. Eng., vol. 8, no. 4, pp. 10809-10814, 2019, doi: 10.35940/ijrte.d4362.118419.

[6] M. N. Meqdad, R. Al-Akam, and S. Kadry, "New prediction method for data spreading in social networks based on machine learning algorithm," TELKOMNIKA Telecommunication, Computing, Electronics and Control, vol. 18, no. 6, pp. 3331-3338, 2020, doi: 10.12928/telkomnika.v18i6.16300.

[7] Z. Rustam and G. S. Saragih, "Prediction schizophrenia using random forest," TELKOMNIKA Telecommunication, Computing, Electronics and Control, vol. 18, no. 3, pp. 1433-1438, 2020, doi: 10.12928/TELKOMNIKA.v18i3.14837.

[8] N. P. Shetty, J. Shetty, R. Narula, and K. Tandona, "Comparison study of machine learning classifiers to detect anomalies," International Journal of Electrical and Computer Engineering, vol. 10, no. 5, pp. 5445-5452, 2020, doi: 10.11591/IJECE.V10I5.PP5445-5452.

[9] M. Vlachos, C. Dunner, R. Heckel, V. G. Vassiliadis, T. Parnell, and K. Atasu, "Addressing Interpretability and Cold-Start in Matrix Factorization for Recommender Systems," IEEE Trans. Knowl. Data Eng., vol. 31, no. 7, pp. 1253-1266, 2019, doi: 10.1109/TKDE.2018.2829521.

[10] R. Saga and Y. Duan, "Apparel Goods Recommender System Based on Image Shape Features Extracted by a CNN," 2018 IEEE International Conference on Systems, Man, and Cybernetics (SMC), 2018, pp. 2365-2369, doi: 10.1109/SMC.2018.00406.

[11] E. G. Nihad, E. N. El Mokhtar, Z. Abdelhamid, and A. A. Mohammed, "Hybrid approach of the fuzzy C-means and the K-nearest neighbors methods during the retrieve phase of dynamic case based reasoning for personalized follow-up of learners in real time," International Journal of Electrical and Computer Engineering, vol. 9, no. 6, pp. 4939-4950, 2019, doi: 10.11591/ijece.v9i6.pp4939-4950.

[12] M. Alzaqebah, N. Alrefai, E. A. E. Ahmed, S. Jawarneh, and M. K. Alsmadi, "Neighborhood search methods with moth optimization algorithm as a wrapper method for feature selection problems," International Journal of Electrical and Computer Engineering, vol. 10, no. 4, pp. 3672-3684, 2020, doi: 10.11591/ijece.v10i4.pp3672-3684. 
[13] S. T. Ahmed, R. Al-Hamdani, and M. S. Croock, "Enhancement of student performance prediction using modified K-nearest neighbor," TELKOMNIKA Telecommunication, Computing, Electronics and Control, vol. 18, no. 4, pp. 1777-1783, 2020, doi: 10.12928/TELKOMNIKA.V18I4.13849.

[14] D. K. Behera, M. Das, S. Swetanisha, and B. Naik, "Collaborative filtering using restricted boltzmann machine and fuzzy C-means," in Advances in Intelligent Systems and Computing, 2018, vol. 710, pp. 723-731, doi: 10.1007/978-981-10-7871-2_69.

[15] R. Salakhutdinov, A. Mnih, and G. Hinton, "Restricted Boltzmann Machines for Collaborative Filtering," in Proceedings of the 24 th International Confer-ence on Machine Learning, 2007, pp. 791-798, doi: 10.1016/j.nonrwa.2008.07.008.

[16] J. Liu, X. Song, Z. Chen, and J. Ma, "Neural fashion experts: I know how to make the complementary clothing matching," Neurocomputing, vol. 359, pp. 249-263, 2019, doi: 10.1016/j.neucom.2019.05.081.

[17] H. M. Ariza, H. H. Martínez, and L. A. Gaviria Roa, "Recognition system for facial expression by processing images with deep learning neural network," Telkomnika (Telecommunication Comput. Electron. Control., vol. 17, no. 6, pp. 2975-2982, 2019, doi: 10.12928/TELKOMNIKA.v17i6.12948.

[18] Q. A. Abed, O. M. Fadhil, and W. L. Al-Yaseen, "Data mining in web personalization using the blended deep learning model," Indonesian Journal of Electrical Engineering and Computer Science, vol. 20, no. 3, pp. 1507-1512, 2020, doi: 10.11591/ijeecs.v20.i3.pp1507-1512.

[19] H. Asil and J. Bagherzadeh, "A new approachto image classification based on adeep multiclass AdaBoosting ensemble," International Journal of Electrical \& Computer Engineering, vol. 10, no. 5, pp. 4872-4880, 2020, doi: 10.11591/ijece.v10i5.pp4872-4880.

[20] C. Li and J. Li, "Collaborative filtering based on dual conditional restricted Boltzmann machines," 2017 36th Chinese Control Conference (CCC), 2017, pp. 10871-10874, doi: 10.23919/ChiCC.2017.8029090.

[21] A. Fischer and C. Igel, "Training restricted Boltzmann machines: An introduction," Pattern Recognition, vol. 47, no. 1, pp. 25-39, 2014, doi: 10.1016/j.patcog.2013.05.025.

[22] L. Ning, R. Pittman, and X. Shen, "LCD: A Fast Contrastive Divergence Based Algorithm for Restricted Boltzmann Machine," Neural Networks, vol. 108, pp. 399-410, 2018, doi: 10.1016/j.neunet.2018.08.018.

[23] A. Pujahari and D. S. Sisodia, "Modeling Side Information in Preference Relation based Restricted Boltzmann Machine for recommender systems," Information Sciences, vol. 490, pp. 126-145, 2019, doi: 10.1016/j.ins.2019.03.064.

[24] Y. Kilani, A. F. Otoom, A. Alsarhan, and M. Almaayah, "A genetic algorithms-based hybrid recommender system of matrix factorization and neighborhood-based techniques," Journal of Computational Science, vol. 28, pp. 78-93, 2018, doi: 10.1016/j.jocs.2018.08.007.

[25] Z. M. Algelal, E. A. G. Aldhaher, D. N. Abdul-Wadood, and R. H. A. Al-Sagheer, "Botnet detection using ensemble classifiers of network flow," International Journal of Electrical and Computer Engineering, vol. 10, no. 3, pp. 2543-2550, 2019, doi: 10.11591/ijece.v10i3.pp2543-2550.

[26] A. C. Alhadi, A. Deraman, M. M. A. Jalil, W. N. J. W. Yussof, and R. Mohemad, "A computational analysis of short sentences based on ensemble similarity model," International Journal of Electrical and Computer Engineering, vol. 9, no. 6, pp. 5386-5394, 2019, doi: 10.11591/ijece.v9i6.pp5386-5394.

[27] A. Alrumaih, A. Al-Sabbagh, R. Alsabah, H. Kharrufa, and J. Baldwin, "Sentiment analysis of comments in social media," International Journal of Electrical \& Computer Engineering (2088-8708), vol. 10, no. 6, pp. 5917-5922, 2020, doi: 10.11591/ijece.v10i6.pp5917-5922.

[28] K. Arun and A. Srinagesh, "Multi-lingual Twitter sentiment analysis using machine learning," International Journal of Electrical \& Computer Engineering, vol. 10, no. 6, pp. 5992-6000, 2020, doi: 10.11591/ijece.v10i6.pp5992-6000.

[29] A. Kumar, J. M. Chatterjee, and V. G. Díaz, "A novel hybrid approach of SVM combined with NLP and probabilistic neural network for email phishing," International Journal of Electrical and Computer Engineering, vol. 10, no. 1, pp. 486-493, 2020, doi: 10.11591/ijece.v10i1.pp486-493.

[30] P. I. Priyadarsini and G. Anuradha, "A novel ensemble modeling for intrusion detection system," International Journal of Electrical and Computer Engineering, vol. 10, no. 2, pp. 1963-1971, 2020, doi: 10.11591/ijece.v10i2.pp1963-1971.

[31] Z. Neili, M. Fezari, and A. Redjati, "ELM and K-nn machine learning in classification of Breath sounds signals," International Journal of Electrical \& Computer Engineering (2088-8708), vol. 10, no. 4, pp. 3528-3536, 2020, doi: 10.11591/ijece.v10i4.pp3528-3536.

[32] D. K. Behera, M. Das, and S. Swetanisha, "Predicting users' preferences for movie recommender system using restricted Boltzmann machine," in Advances in Intelligent Systems and Computing, 2019, vol. 711, pp. 759-769, doi: 10.1007/978-981-10-8055-5_67.

[33] X. Bai, M. Wang, I. Lee, Z. Yang, X. Kong, and F. Xia, "Scientific paper recommendation: A survey," IEEE Access, vol. 7, pp. 9324-9339, 2019, doi: 10.1109/ACCESS.2018.2890388.

[34] Y. Koren and J. Sill, "Collaborative filtering on ordinal user feedback," Twenty-third international joint conference on artificial intelligence, pp. 3022-3026, 2013.

[35] Q. Wang, B. Peng, X. Shi, T. Shang, and M. Shang, "DCCR: Deep Collaborative Conjunctive Recommender for Rating Prediction," IEEE Access, vol. 7, pp. 60186-60198, 2019, doi: 10.1109/ACCESS.2019.2915531.

[36] A. A. Ojugo and D. O. Otakore, "Computational solution of networks versus cluster grouping for social network contact recommender system," International Journal of Information and Communication Technology, vol. 9, no. 3, pp. 185-194, 2020, doi: 10.11591/ijict.v9i3.pp185-194. 


\section{BIOGRAPHIES OF AUTHORS}
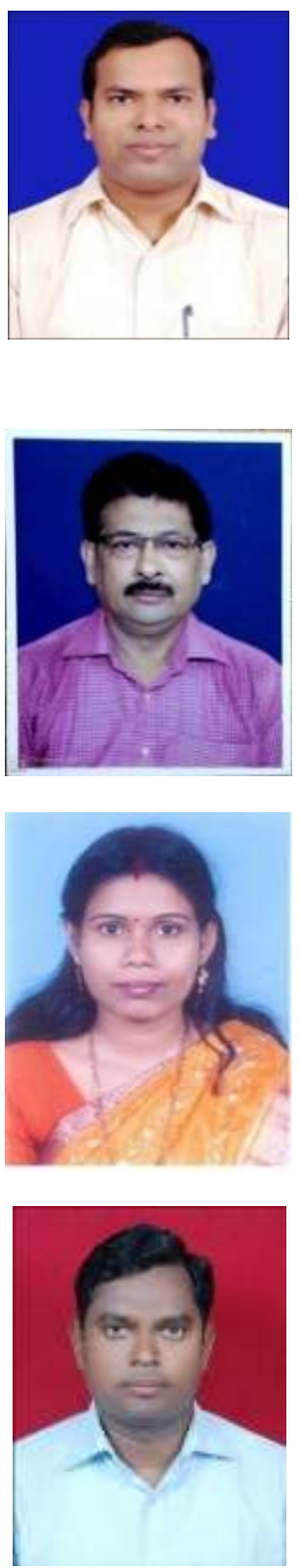

Dayal Kumar Behera has obtained B.E. degree with honours in Information Technology from National Institute of Science and Technology, Berhampur, Odisha in the year 2006 and completed M.Tech., from College of Engineering and Technology, Bhubaneswar, in 2012. Currently he is pursuing his Ph.D. at KIIT University, Odisha. He has been working as an Asst. Professor in the department of CSE at Silicon Institute of Technology, Bhubaneswar. He is having thirteen years of teaching experience and carrying out research in various aspects of Recommender System, Machine Learning, Data Science, IoT and Remote Sensing. He is having many publications in various journals and conferences and guided many B.Tech. and M.Tech. projects in his area of interests. He is a life member of Indian Society for Technical Education (ISTE) and IAENG.was the Editor-in-Chief of the IEEE Transactions on Power Electronics from 2006 to 2012.

Madhabananda Das has been working as a Senior Professor in School of Computer Engineering, KIIT Deemed to be University, Bhubaneswar, Odisha. He is having 26 years of teaching experience and 13 years of industry experience. His research interests encompass Computational Intelligence, Soft Computing, Artificial Intelligence and pattern recognition. $\mathrm{He}$ is having a large number of research publications in various international conference proceedings and journals and guided many M.Tech. and Ph.D. Scholars in his areas of interest.

Subhra Swetanisha has been working an Assistant Professor with the Department of Computer Science and Engineering in Trident Academy of Technology Bhubaneswar, Odisha, India. She has received M.Tech. degree in Computer Science and Engineering from KIIT University in 2009 and completed B.E. from Utkal University in 2005. Currently she is working as a Ph.D. scholar in KIIT University, India. Her current research interests include Machine Learning, Data Science and Remote Sensing. She is having fourteen years of professional experience and published many research papers in various journals and conferences. She is a life member of Indian Society for Technical Education (ISTE) and IAENG.

P. K. Sethy currently working as Assistant Professor in Department of Electronics, Sambalpur University since 2013 . He has 8 years of teaching, research \& administrative experience and 4 years of Industry experience. Previously he worked as Engineer in Doordarshan, Prashar Bharati since 2009 to 2013. He has received his Ph.D. and M. Tech degree from Sambalpur University and IIT (ISM) Dhanbad respectively. His research area is image processing, machine learning and deep learning. He has published 60 research paper in different repute journal and conference. In addition, he has two patents. He is also editorial board member of International Journal of Electrical and Computer Engineering. Recently he has received "InSc Young Achiever Award" for the research paper "Detection of coronavirus (COVID-19) based on Deep Features and Support Vector Machine, organized by Institute of Scholars, Ministry of MSME, Government of India. He is Senior Member of IEEE. He is the frequent reviewer of Journal of Computational and Theoretical Nanoscience, Journal of Intelligent \& Fuzzy Systems, Indonesian Journal of Electrical Engineering and Informatics, TELKOMNIKA Telecommunication, Computing, Electronics and Control, Karbala International Journal of Modern Science, Journal of Ambient Intelligence and Humanized Computing, Scientific African, ACM Transactions on Computational Biology and Bioinformatics, Current Medical Imaging, Journal of Computational and Theoretical Nanoscience, Concurrent Engineering: Research and Applications, Information Security Journal: A Global Perspective, Journal of XRay Science and Technology, Asian Journal of Medical Principles and Clinical Practice, IEEE Access, Chaos, Solitons \& Fractals, Spanish Journal of Agricultural Research, Computers in Biology and Medicines, Multimedia Tools and Applications, International Journal of TROPICAL DISEASE \& Health, Journal of Electronic Imaging, International Journal of Speech Technology, Springer, AI and Society, Computer and Electronics in Agriculture, Journal of Food Process Engineering,Journal of Supercomputing, Biomedical Signal Processing \& Control, Journal of Applied Research on Medicinal and Aromatic Plants, Scientific Report, International Journal of System Assurance Engineering and Management, Wireless Personal Communications. 